\title{
Convergence of numerical solutions for a class of stochastic age-dependent capital system with fractional Brownian motion
}

\author{
Lai-Yun Zhenga , Qi-Min Zhang \\ a School of Mechanical Engineering, Ningxia University, Yinchuan 750021, China. \\ ${ }^{b}$ School of Mathematics and Statistics, Ningxia University, Yinchuan 750021, China.
}

Communicated by A. Atangana

\begin{abstract}
In this paper, we consider a class of stochastic age-dependent capital system with fractional Brownian motion, and investigate the convergence of numerical approximate solution. It is proved that the numerical approximation solutions converge to the analytic solutions of the equations under given conditions. A numerical example is provided to illustrate the theoretical results. (C)2017 All rights reserved.
\end{abstract}

Keywords: Stochastic age-dependent capital system, numerical solution, Euler approximation, fractional Brownian motion. 2010 MSC: 65C35, 60G22, 65M12.

\section{Introduction}

Stochastic partial differential equations have been widely used to model the phenomena arising in many fields of science and industry such as finance, economics, biology and medicine, see e.g., $[4,6,12,13,19,21]$ and references therein. Recently, the numerical construction of stochastic agedependent (vintage) capital system with standard Brownian motion has captured many researchers' attention. For example, Zhang et al. [21,22] discussed the exponential stability of numerical solutions for the stochastic age-dependent capital system with Poisson jumps in the case of deterministic magnitude, and further studied the convergence of numerical solutions for a class of stochastic age-dependent capital system with random jump magnitudes. In their subsequent work [20], they constructed a split-step backward Euler method for stochastic age-dependent capital system with Markovian switching and proved that, under the one-sided local Lipschitz condition on the drift and local Lipschitz condition on the diffusion, the split-step backward Euler method converges with strong order of one half to the true solution. Du et al. [2] discussed the convergence of a semi-implicit Euler method for stochastic age-dependent capital system with variable delays and random jump magnitudes. In these studies, uncertainties in the financial market (i.e., randomness of the external environment) were considered in their models in the

\footnotetext{
*Corresponding author

Email addresses: zhenglaiyun@126.com (Lai-Yun Zheng), zhangqimin64@sina.com (Qi-Min Zhang)
} 
form of a standard Brownian motion. However, the randomness of the external environment is not always well modeled by the standard Brownian motion (e.g., because of the long-range dependence or long memory of the processes under consideration). In the last few years, some articles have been published choosing fractional Brownian motion (fBm) as an underlying diffusive process (e.g., Refs. [7-9, 14-17] and references therein). For example, Jiang et al. [9] proposed a class of stochastic heat equations with first order fractional noises and established the existence and uniqueness of the solution of the equation. Wang et al. [15] studied the problem of continuous time option pricing with transaction costs by using the homogeneous subdiffusive $\mathrm{fBm}$ as a model of asset prices. Xiao et al. [17] presented a pricing model for equity warrants in a mixed fractional Brownian environment and proposed a hybrid intelligent algorithm to solve the nonlinear optimization problem. Jańczak-Borkowska [8] investigated the existence and uniqueness of generalized backward stochastic differential equation driven by $\mathrm{fBm}$ with Hurst parameter $\mathrm{H}$ greater than $1 / 2$, and shown the connection between this solution and the solution of parabolic partial differential equation with Neumann boundary condition. Rostek and Schöbel [14] gave a note on the use of $\mathrm{fBm}$ for financial modeling and clarified that $\mathrm{fBm}$ is suited for economic modeling. In this paper, motivated by [14], we consider a class of stochastic age-dependent capital system with $\mathrm{fBm}$ as following

$$
\begin{cases}\frac{\partial K(a, t)}{\partial t}+\frac{\partial K(a, t)}{\partial a}=-\mu(a, t) K(a, t)+f(t, K(a, t))+g(t, K(a, t)) \frac{d B_{t}^{H}}{d t}, & i n Q \\ N(t)=\int_{0}^{A} K(a, t) d a, & t \in[0, T] \\ K(0, t)=\phi(t)=\gamma(t) A(t) F(L(t), N(t)), & t \in[0, T] \\ K(a, 0)=K_{0}(a), & a \in[0, A]\end{cases}
$$

where $\mathbf{Q}=(0, A) \times(0, T), K(a, t)$ is the stock of capital goods of age a at time $t$, and $N(t)$ is the total sum of the capital. The investment $\phi(t)$ in the new capital and $f(t, K(a, t))$ in the capital of age $a$ are the endogenous (unknown) variables. The maximum physical lifetime of capital $A$, the planning interval of calendar time $[0, T]$, the depreciation rate $\mu(a, t)$ of capital, and the capital density $K_{0}(a)$ (the initial distribution of capital over age) are given. $\gamma(t)(\in(0,1))$ and $A(t)$ denote the accumulative rate and the technical progress at the moment of $t$, respectively. $F(L(t), N(t))$ is the production function and $L(t)$ is the labor force. $f(t, K(a, t))+g(t, K(a, t)) \frac{\partial B_{t}^{H}}{\partial t}$ denotes effects of the external environment for the system, such as innovations in techniques, introduction of new products, natural disasters and changes in laws and government policies and so on. The effects of external environment include the deterministic and the random parts which depend on $t$ and $K(a, t)$. Here $B_{t}^{H}$ is standard $f B m$ with Hurst parameter $H \in\left(\frac{1}{2}, 1\right)$. Fractional Brownian motion has $\mathrm{H}$-self-similarity, in addition it has long-range dependence when $\mathrm{H}>\frac{1}{2}$ and short-range dependence when $\mathrm{H}<\frac{1}{2}$. When $\mathrm{H}=\frac{1}{2}$, the corresponding $\mathrm{fBm}$ is a standard Brownian motion and has independent increments. The standard Brownian motion is a semimartingales if and only if $\mathrm{H}=\frac{1}{2}$, thus Itô stochastic integration theory for semimartingales cannot be applied if $\mathrm{H} \neq \frac{1}{2}$.

The system (1.1) describes the evolution of the composition of the productive capital as a function of purchasing/selling new or used capital. When $g \equiv 0$ (i.e., without stochastically perturbed), $K(a, t)$ denotes the riskless capital and the system becomes to a generalization of the deterministic age-dependent capital system, which has been studied by many authors. For instance, Feichtinger et al. [4, 5] established deterministic (vintage) capital dynamics and the necessary optimality conditions, and developed the vintage capital stock model with technological progress while the model is solved. Goetz et al. [6] studied the capital replacement decision of a firm as a distributed investment and disinvestment optimal control problem.

Due to the nonlinear feature of stochastic system with $\mathrm{fBm}$, and its dificullty to bring out explicit solutions, constructing the efficient computational methods becomes extremely important. Thus numerical approximation schemes are invaluable tools for exploring their properties. Numerical solution of stochastic differential equations (SDEs) driven by Brownian motion has been studied by many researchers. For instance, Kloeden et al. [10] have given some useful results in their monograph, which contains an almost complete survey of the theory of numerical solution of such SDEs with regular coefficients. Zhang [18] discussed the existence and uniqueness for stochastic age-dependent population equation with dif- 
fusion. Ronghua et al. [13] studied the convergence of numerical solutions to stochastic age-dependent population equations with diffusions and Markovian switching. Recently, Ma et al. [11] studied the stochastic age-dependent population equations with fractional Brownian motion and developed a numerical scheme and showed the convergence of the numerical approximation solution to the analytic solution. Rathinasamy [12] constructed a class of split-step $\theta$-methods for solving stochastic age-dependent population equations with Markovian switching. The main aim of this paper is to investigate the convergence of numerical approximation for a class of stochastic age-dependent capital system with fBm under the given conditions.

The rest of this paper is organized as follows. In Section 2 some basic preliminaries results, which are essential for our development, and the Euler approximation analysis are introduced. The main results are shown in Section 3. In Section 4 a numerical example is provided to illustrate the theoretical results.

\section{Preliminaries and approximation}

Definition 2.1 (fractional Brownian motion $(\mathrm{fBm})$ ). Let $\mathrm{B}^{\mathrm{H}}=\mathrm{B}^{\mathrm{H}}(\mathrm{t}), \mathrm{t} \in \mathrm{R}$ be a stochastic process, and $0<\mathrm{H}<1$. $\mathrm{B}_{\mathrm{t}}^{\mathrm{H}}$ is called a $\mathrm{fBm}$ with Hurst parameter $\mathrm{H}$, if it is a centered Gaussian process, which starts at zero $\left(B_{0}^{H}=0\right)$, has expectation zero for all $t$, i.e., $E\left[B_{t}^{H}\right]=0$, and has the covariance function

$$
E\left[B_{t}^{H} B_{s}^{H}\right]=\frac{1}{2}\left(|t|^{2 H}+|s|^{2 H}-|t-s|^{2 H}\right), \quad t, s \in R .
$$

Let

$$
\mathrm{V}=\mathbb{H}^{1}([0, A]) \equiv\left\{\varphi \mid \varphi \in \mathrm{L}^{2}([0, A]), \frac{\partial \varphi}{\partial x_{i}} \in \mathrm{L}^{2}([0, A]), \text { where } \frac{\partial \varphi}{\partial x_{i}} \text { are generalized partial derivatives }\right\} .
$$

$V$ is a Sobolev space. $\mathbb{H}=\mathrm{L}^{2}([0, A])$ such that

$$
\mathrm{V} \hookrightarrow \mathbb{H} \equiv \mathbb{H}^{\prime} \hookrightarrow \mathrm{V}^{\prime} .
$$

Then $\mathrm{V}^{\prime}=\mathbb{H}^{-1}([0, \mathrm{~A}])$ is the dual space of $\mathrm{V}$. We denote by $|\cdot|$ and $\|\cdot\|$ the norms in $\mathrm{V}$ and $\mathrm{V}^{\prime}$, respectively, by $\langle\cdot, \cdot\rangle$ the duality product between $\mathrm{V}, \mathrm{V}^{\prime}$, and by $(\cdot, \cdot)$ the scalar product in $\mathbb{H}$. $\mathrm{m}$ is a constant such that

$$
\|x\| \leqslant \mathrm{m}\|x\|, \forall x \in \mathrm{V} .
$$

Throughout this paper, $f(t, \cdot): V \rightarrow V^{\prime}$ is a family of nonlinear operators a.e.t for all $t . g(t, \cdot): V \rightarrow$ $\mathcal{L}(\mathrm{K}, \mathrm{H})$ is the family of all bounded linear operators from $\mathrm{K}$ into $\mathrm{H}$. Let $\mathrm{C}=\mathrm{C}([0, \mathrm{~T}] ; \mathrm{H})$ be the space of all continuous functions from $[0, T]$ into $\mathbb{H}$ with sup-norm $\|\psi\|_{C}=\sup _{0 \leqslant s \leqslant T}|\psi(s)|, L_{V}^{p}=L^{p}([0, T] ; V)$ and $L_{\mathbb{H}}^{p}=$ $\mathrm{L}^{\mathfrak{p}}([0, \mathrm{~T}] ; \mathbb{H})$. We assume that there exists a unique process $\mathrm{K}(\mathrm{a}, \mathrm{t}) \in \mathrm{I}^{\mathfrak{p}}([0, \mathrm{~T}] ; \mathrm{V}) \cap \mathrm{L}^{2}=\mathrm{L}^{\mathrm{p}}(\omega, \mathrm{C}([0, \mathrm{~T}] ; \mathbb{H}))$ such that Eq. (1.1) holds.

The integral version of Eq. (1.1) is given as follows

$$
\begin{cases}K_{t}=K_{0}-\int_{0}^{t} \frac{\partial K_{s}}{\partial a} d s-\int_{0}^{t} \mu(a, s) d s+\int_{0}^{t} f\left(s, K_{s}\right) d s+\int_{0}^{t} g\left(s, K_{s}\right) d B_{s}^{H}, & t \in[0, T], \\ K(0, t)=\phi(t)=\gamma(t) A(t) F\left(L(t), \int_{0}^{A} K(a, t) d a\right), & t \in[0, T],\end{cases}
$$

where $K_{t}=K(a, t)$.

For system (1.1) the discrete Euler approximate solution on $t=0, h, 2 h, \cdots, N h$ is defined by the following iterative scheme

$$
Q_{t}^{k+1}=Q_{t}^{k}-\frac{\partial Q_{t}^{k}}{\partial a} h-\mu\left(a, t_{k}\right) Q_{t}^{k} h+f\left(t_{k}, Q_{t}^{k}\right) h+g\left(t_{k}, Q_{t}^{k}\right) \Delta B_{t}^{H},
$$

with initial value $Q_{t}^{0} \equiv K(a, 0), Q^{k}(0, t) \equiv \gamma(t) A(t) F\left(L(t), \int_{0}^{A} Q_{t}^{k} d a\right)$. Where $Q_{t}^{k}$ is an approximation to $\mathrm{K}\left(\mathrm{a}, \mathrm{t}_{\mathrm{k}}\right)$ for $\mathrm{t}_{\mathrm{k}}=k \mathrm{~h}, \mathrm{~h}=\Delta \mathrm{t}=\mathrm{T} / \mathrm{N} \ll 1$ is the time increment, and $\Delta \mathrm{B}_{\mathrm{t}}^{\mathrm{H}}=\mathrm{B}_{\mathrm{t}+\Delta \mathrm{t}}^{\mathrm{H}}-\mathrm{B}_{\mathrm{t}}^{\mathrm{H}}$ is fBm increment. 
For convenience, we shall extend the discrete numerical solution (2.2) to continuous time,

$$
Q_{t}=K_{0}-\int_{0}^{t} \frac{\partial Z_{s}}{\partial a} d s-\int_{0}^{t} \mu(a, s) Z_{s} d s+\int_{0}^{t} f\left(s, Z_{s}\right) d s+\int_{0}^{t} g\left(s, Z_{s}\right) d B_{s}^{H},
$$

where we have defined the piecewise constant function

$$
Z_{t}=Z(a, t) \equiv \sum_{k=0}^{N-1} Q_{t}^{k} I_{[k h,(k+1) h)}(t)
$$

and $\mathbf{I}_{G}$ is the indicator function for the set $G$. It is straightforward to check that $Z\left(a, t_{k}\right)=Q_{t}^{k}=Q\left(a, t_{k}\right)$. For convenience, the expression (2.3) may also be expressed in the stochastic differential form

$$
d Q_{t}=-\frac{\partial Z_{t}}{\partial a} d t-\mu(a, t) Z_{t} d t+f\left(t, Z_{t}\right) d t+g\left(t, Z_{t}\right) d B_{t}^{H}
$$

Let $\mathrm{G}$ be an open subset of $\mathrm{V}$, and $\mathcal{D} \subseteq \mathrm{G}$ be any compact set. We denote the unique solution of Eq. (1.1) for all $t \in[0, T]$ by $K(a, t) \in G$ with initial condition $K_{0} \in G$, and the Euler approximation solution to Eq. (1.1) by $Q_{t}^{k}$.

As the standing hypotheses we always assume that the following conditions are satisfied:

(a) $\mu(a, t)$ is non-negative measurable in $\mathbf{Q}$, and $\gamma(t)$ and $A(t)$ are nonnegative continuous in $[0, T]$ such that

$$
\begin{cases}0 \leqslant \mu_{0} \leqslant \mu(a, t) \leqslant \bar{\mu}<\infty, & (a, t) \in Q \\ \gamma(t) A(t) \leqslant \eta, \eta \text { is a non-negative constant, } & \text { in }[0, T]\end{cases}
$$

where $\int_{0}^{A} \mu(a, t) d a=+\infty . F(L, N)$ is satisfied with

$$
\left\{\begin{array}{l}
F(L, N) \geqslant 0(F(L, 0)=0), \quad \frac{\partial F}{\partial L}>0, \\
0<\frac{\partial F}{\partial N}<F_{1}, \text { where } F_{1} \text { is a positive constant. }
\end{array}\right.
$$

(b) (Lipschitz condition). There exists a positive constant $m_{1}(\mathcal{D})$ for all $K_{1}, K_{2} \in \mathcal{D}$ such that

$$
\left|f\left(t, K_{2}\right)-f\left(t, K_{1}\right)\right|^{2} \vee\left\|g\left(t, K_{2}\right)-g\left(t, K_{1}\right)\right\|_{2}^{2} \leqslant m_{1}(\mathcal{D})\left|K_{2}-K_{1}\right|^{2} .
$$

(c) There exists a $C^{1,2}$-positive function $V(t, \cdot): G \rightarrow R_{+}$, such that $K \in G: V(t, K) \leqslant r$ is compact for any $r>0$.

(d) Let $\psi_{1}(t), \psi_{2}(t)$ be two continuous non-negative functions, and there exists a positive constant $m_{2}(\mathcal{D})$. Assume that for all $\mathrm{K} \in \mathrm{G}$,

$$
L V(t, K) \leqslant m_{2}(\mathcal{D})+\psi_{1}(t)+\psi_{2}(t) V(t, K)
$$

where

$$
\begin{aligned}
\int_{0}^{T} L V(t, K) d t \equiv & \int_{0}^{T}\left(V_{t}(t, K)+\left\langle V_{K}(t, K),-\frac{\partial K}{\partial a}-\mu(a, t) K+f(t, K)\right\rangle\right) d t \\
& +\frac{1}{2} H \int_{0}^{T} t^{2 H-1} V_{K K}(t, K)\|g(t, K)\|_{2}^{2} d t .
\end{aligned}
$$

(e) There exists a positive constant $m_{3}(\mathcal{D})$ such that for all $\mathrm{K}_{1}, \mathrm{~K}_{2} \in \mathcal{D}$

$$
\begin{aligned}
& \left|V\left(t, K_{1}\right)-V\left(t, K_{2}\right)\right| V\left|V_{t}\left(t, K_{1}\right)-V_{t}\left(t, K_{2}\right)\right| V\left|V_{K}\left(t, K_{1}\right)-V_{K}\left(t, K_{2}\right)\right| V\left|V_{K K}\left(t, K_{1}\right)-V_{K K}\left(t, K_{2}\right)\right| \\
& \quad \leqslant m_{3}(\mathcal{D})\left|K_{1}-K_{2}\right| .
\end{aligned}
$$

Remark 2.2. When $\mathrm{H}=\frac{1}{2}$, the $\mathrm{fBm}$ is a standard Brownian motion. 
If condition (b) holds, there exists a positive constant $m_{4}(\mathcal{D})$ such that

$$
|f(t, K)|^{2} \vee\|g(t, K)\|_{2}^{2} \leqslant m_{4}(\mathcal{D})
$$

for all $K \in \mathcal{D}$. Since $K(a)$ is bounded, there exists a positive constant $m_{5}(\mathcal{D})$ such that

$$
|K(a, t)|^{2} \leqslant m_{5}(\mathcal{D}) .
$$

Since $K(a, t) \in V=\mathbb{H}^{1}([0, A]), \frac{\partial}{\partial a}: V \rightarrow V^{\prime}$ is a family of linear operators, then there also exists a positive constant $\mathrm{m}_{6}(\mathcal{D})$ such that

$$
\left|\frac{\partial K}{\partial a}\right|^{2} \leqslant m_{6}(\mathcal{D})
$$

for $K \in \mathcal{D}$.

\section{Main results}

In this section, we will give and prove the following main results that the numerical solutions converge to the true ones of the stochastic age-dependent capital equations with respect to $\mathrm{fBm}$ under the given conditions.

Theorem 3.1. If $\tau$ is the first exit time of the solution $\mathrm{K}(\mathrm{a}, \mathrm{t})$ or the Euler approximate solution $\mathrm{Q}_{\mathrm{t}}$ in a bounded region $\mathcal{D}$, and for all $a \in(0, A), \sup _{t \in \mathcal{D}}\left|\frac{Q_{t}-K(a, t)}{Z_{t}-K(a, t)}\right|$ exists, and $f(t, K)$ and $g(t, K)$ satisfy condition $(b)$, then for $\Delta \mathrm{tT}<1$

$$
E\left[\sup _{0 \leqslant \mathrm{t} \leqslant \tau \wedge T}\left|\mathrm{Q}_{\mathrm{t}}-\mathrm{K}(\mathrm{a}, \mathrm{t})\right|^{2}\right] \leqslant \mathrm{C}_{1}(\mathcal{D}) e^{\mathrm{C}_{2}(\mathcal{D}) \mathrm{T}} \Delta \mathrm{t}=\mathrm{C}(\mathcal{D}) \Delta \mathrm{t}
$$

where

$$
\begin{aligned}
\mathrm{C}_{1}(\mathcal{D})= & 8\left\{\left[\mathrm{AF}_{1}^{2} \eta^{2} \mathrm{M}(\mathcal{D})+\mu_{0}+\mathrm{m}_{1}(\mathcal{D})\left(1+2 \mathrm{HT}_{1}^{2 \mathrm{H}-1}\right)\right] *\left[\mathrm{~m}_{6}(\mathcal{D})+\mu_{0}^{2} \mathrm{~m}_{5}(\mathcal{D})+\mathrm{m}_{4}(\mathcal{D})+\mathrm{m}_{4}(\mathcal{D}) \mathrm{T}_{1}^{2 \mathrm{H}}\right]\right. \\
& \left.+\mathrm{m}_{4}^{\frac{1}{2}}(\mathcal{D}) \mathrm{m}_{5}^{\frac{1}{2}}(\mathcal{D}) \mathrm{L}_{1} \mathrm{~L}_{2}^{\mathrm{H}}\right\} \\
\mathrm{C}_{2}(\mathcal{D})= & 2 A \mathrm{~F}_{1}^{2} \eta^{2} \mathrm{M}(\mathcal{D})+3 \mu_{0}+1+2 \mathrm{~m}_{1}(\mathcal{D})\left(1+2 \mathrm{HT}_{1}^{2 \mathrm{H}-1}\right) .
\end{aligned}
$$

Thus, the Euler approximation solution $\mathrm{Q}_{\mathrm{t}}$ converges to the unique exact solution $\mathrm{K}(\mathrm{a}, \mathrm{t})$ when $\Delta \mathrm{t} \rightarrow 0$ as long as $\mathrm{Q}_{\mathrm{t}}$ and $\mathrm{K}(\mathrm{a}, \mathrm{t})$ remain within the domain $\mathcal{D}$.

Proof. We consider one and only trajectories $K(a, t)$ and $Q_{t}$ which remain within a bounded domain $\mathcal{D}$. Firstly, we introduce the stopping time $\tau=\rho \wedge \theta$ where

$$
\rho=\inf \left\{t \geqslant 0: Q_{t} \bar{\in} \mathcal{D}\right\} \text { and } \theta=\inf \{t \geqslant 0: K(a, t) \bar{\in} \mathcal{D}\}
$$

are the first exit time that $Q_{t}$ and $K(a, t)$ leave from the domain $\mathcal{D}$, respectively.

The bounded domain $\mathcal{D}$ is defined as

$$
\mathcal{D}=\mathcal{D}(\mathrm{r}) \equiv\{\mathrm{K} \in \mathrm{G} \text { such that } \mathrm{V}(\mathrm{t}, \mathrm{K}) \leqslant \mathrm{r}\} .
$$

Applying Itô formula to $\left|Q_{t}-K(a, t)\right|^{2}$ yields

$$
\begin{aligned}
\left|Q_{t}-K(a, t)\right|^{2}= & 2 \int_{0}^{t}\left\langle-\frac{\partial Z_{s}}{\partial a}+\frac{\partial K(a, s)}{\partial a}-\mu(a, s)\left(Z_{s}-K(a, s)\right), Q_{s}-K(a, s)\right\rangle d s \\
& +2 \int_{0}^{t}\left(f\left(s, Z_{s}\right)-f(s, K(a, s)), Q_{s}-K(a, s)\right) d s+2 H \int_{0}^{t} s^{2 H-1}\left\|g\left(s, Z_{s}\right)-g(s, K(a, s))\right\|_{2}^{2} d s
\end{aligned}
$$




$$
\begin{aligned}
& +2 \int_{0}^{t}\left(Q_{s}-K(a, s), g\left(s, Z_{s}\right)-g(s, K(a, s))\right) d B_{s}^{H} \\
\leqslant & -2 \int_{0}^{t}\left\langle\frac{\partial\left(Z_{s}-K(a, s)\right)}{\partial a}, Q_{s}-K(a, s)\right\rangle d s-2 \mu_{0} \int_{0}^{t}\left(Z_{s}-K(a, s), Q_{s}-K(a, s)\right) d s \\
& +2 \int_{0}^{t}\left(f\left(s, Z_{s}\right)-f(s, K(a, t)), Q_{s}-K(a, s)\right) d s+2 H \int_{0}^{t} s^{2 H-1}\left\|g\left(s, Z_{s}\right)-g(s, K(a, s))\right\|_{2}^{2} d s \\
& +2 \int_{0}^{t}\left(Q_{s}-K(a, s), g\left(s, Z_{s}\right)-g(s, K(a, s))\right) d B_{s}^{H} .
\end{aligned}
$$

Since

$$
\begin{aligned}
& -\left\langle\frac{\partial\left(Z_{t}-K(a, t)\right)}{\partial a}, Q_{t}-K(a, t)\right\rangle \\
& =-\int_{0}^{A} \frac{\partial\left(Z_{t}-K(a, t)\right)}{\partial a}\left(Q_{t}-K(a, t)\right) d a \\
& =-\int_{0}^{A} \frac{\partial\left(Z_{t}-K(a, t)\right)}{\partial a}\left(Z_{t}-K(a, t)\right) \frac{Q_{t}-K(a, t)}{Z_{t}-K(a, t)} d a \\
& =-\int_{0}^{A} \frac{Q_{t}-K(a, t)}{Z_{t}-K(a, t)} \frac{\partial\left(Z_{t}-K(a, t)\right)}{\partial a} d_{a}\left(Z_{t}-K(a, t)\right) \\
& \leqslant \frac{1}{2} \gamma^{2}(s) A^{2}(s)\left[F\left(L(s), \int_{0}^{A} Z_{t}-K(a, t) d a\right)-F(L(s), 0)\right]_{t \in \mathcal{D}}^{2} \sup _{t}\left|\frac{Q_{t}-K(a, t)}{Z_{t}-K(a, t)}\right| d a \\
& \leqslant \frac{1}{2} M(\mathcal{D}) \eta^{2}\left(\left.\frac{\partial F(L, N)}{\partial N}\right|_{y}\right)^{2}\left(\int_{0}^{A} Z_{t}-K(a, t) d a\right) \\
& \leqslant \frac{1}{2} A F_{1}^{2} \eta^{2} M(\mathcal{D})\left|Z_{t}-K(a, t)\right|^{2},
\end{aligned}
$$

where $M(\mathcal{D})=\sup _{t \in \mathcal{D}}\left|\frac{Q_{t}-K(a, t)}{Z_{t}-K(a, t)}\right|$ for all $a$, therefore, we get that

$$
\begin{aligned}
\left|Q_{t}-K(a, t)\right|^{2} \leqslant & A F_{1}^{2} \eta^{2} M(\mathcal{D})\left|Z_{t}-K(a, t)\right|^{2} \\
& +2 \mu_{0} \int_{0}^{t}\left|Z_{s}-K(a, s)\left\|Q_{s}-K(a, s)\left|d s+2 \int_{0}^{t}\right| Q_{s}-K(a, s)\right\| f\left(s, Z_{s}\right)-f(s, K(a, t))\right| d s \\
& +2 H \int_{0}^{t} s^{2 H-1}\left\|g\left(s, Z_{s}\right)-g(s, K(a, s))\right\|_{2}^{2} d s+2 \int_{0}^{t}\left(Q_{s}-K(a, s), g\left(s, Z_{s}\right)-g(s, K(a, s))\right) d B_{s}^{H} \\
\leqslant & A F_{1}^{2} \eta^{2} M(\mathcal{D})\left|Z_{t}-K(a, t)\right|^{2}+\mu_{0} \int_{0}^{t}\left|Z_{s}-K(a, s)\right|^{2}+\mu_{0} \int_{0}^{t}\left|Q_{s}-K(a, s)\right|^{2} d s \\
& +\int_{0}^{t}\left|Q_{s}-K(a, s)\right|^{2}+\int_{0}^{t}\left|f\left(s, Z_{s}\right)-f(s, K(a, t))\right|^{2} d s+2 H \int_{0}^{t} s^{2 H-1} \| g\left(s, Z_{s}\right) \\
& -g(s, K(a, s)) \|_{2}^{2} d s+2 \int_{0}^{t}\left(Q_{s}-K(a, s), g\left(s, Z_{s}\right)-g(s, K(a, s))\right) d B_{s}^{H} .
\end{aligned}
$$

Since the coefficients of Eq. (1.1) are Lipschitz continuous and $K(a, s)$ and $Q_{s}$ are bounded, we get that

$$
\mid f\left(s, Z_{s}\right)-f\left(s,\left.K(a, s)\right|^{2} \vee \| g\left(s, Z_{s}\right)-g\left(s, K(a, s) \|_{2}^{2} \leqslant m_{1}(\mathcal{D})\left|Z_{s}-K(a, s)\right|^{2}\right.\right.
$$

for $s \in\left[0, \tau \wedge T_{1}\right]$. Let $T_{1} \in[0, T]$ be an arbitrary time. For any $t \in\left[0, \tau \wedge T_{1}\right]$, we have 


$$
\begin{aligned}
\int_{0}^{\tau \wedge T_{1}} s^{2 H-1}\left\|g\left(s, Z_{s}\right)-g(s, K(a, s))\right\|^{2} d s & \leqslant m_{1}(\mathcal{D}) \int_{0}^{\tau \wedge T_{1}} s^{2 H-1}\left|Z_{s}-K(a, s)\right|^{2} d s \\
& \leqslant m_{1}(\mathcal{D}) \sup _{0 \leqslant s \leqslant \tau \wedge T_{1}} s^{2 H-1} \int_{0}^{\tau \wedge T_{1}}\left|Z_{s}-K(a, s)\right|^{2} d s \\
& \leqslant m_{1}(\mathcal{D}) T_{1}^{2 H-1} \int_{0}^{\tau \wedge T_{1}}\left|Z_{s}-K(a, s)\right|^{2} d s .
\end{aligned}
$$

Therefore,

$$
\begin{aligned}
E\left(\sup _{0 \leqslant t \leqslant \tau \wedge T_{1}}\left|Q_{t}-K(a, t)\right|^{2}\right) \leqslant & {\left[A F_{1}^{2} \eta^{2} M(\mathcal{D})+\mu_{0}+m_{1}\left(1+2 H T_{1}^{2 H}-1\right)\right] \int_{0}^{\tau \wedge T_{1}} E\left|Z_{s}-K(a, s)\right|^{2} d s } \\
& +\left(\mu_{0}+1\right) \int_{0}^{\tau \wedge T_{1}} E\left|Q_{s}-K(a, s)\right|^{2} d s \\
& +2 E \sup _{0 \leqslant t \leqslant \tau \wedge T_{1}} \int_{0}^{t}\left(Q_{s}-K(a, s), g\left(s, Z_{s}\right)-g(s, K(a, s))\right) d B_{s}^{H} .
\end{aligned}
$$

By the basic inequality $a^{2}+b^{2} \geqslant 2 a b$ and an Itô formula for a finite dimensional fractional Brownian motion applied to $(K(t, a), t \in[0, T])$ (e.g., [3]), we can get

$$
\begin{aligned}
& E \sup _{0 \leqslant t \leqslant \tau \wedge T_{1}} \int_{0}^{t}\left(Q_{s}-K(a, s), g\left(s, Z_{s}\right)-g(s, K(a, s))\right) d B_{s}^{H} \\
& \leqslant E \int_{0}^{\tau \wedge T_{1}}\left|Q_{s}-K(a, s)\right|\left\|g\left(s, Z_{s}\right)-g(s, K(a, s))\right\|_{2} d B_{s}^{H} \\
& \leqslant 2 m_{5}^{\frac{1}{2}}(\mathcal{D}) L_{1}\left[E\left(\int_{0}^{\tau \wedge T_{1}}\left\|g\left(s, Z_{s}\right)-g(s, K(a, s))\right\|_{2} d B_{s}^{H}\right)^{2}\right]^{\frac{1}{2}} \\
& \leqslant 4 m_{4}^{\frac{1}{2}}(\mathcal{D}) m_{5}^{\frac{1}{2}}(\mathcal{D}) L_{1} L_{2}^{H} \Delta t
\end{aligned}
$$

where $L_{1}$ and $L_{2}$ are two positive constants. Thus, it follows from (3.1) and (3.2)

$$
\begin{aligned}
& E\left(\sup _{0 \leqslant \mathrm{t} \leqslant \tau \wedge T_{1}}\left|Q_{t}-K(a, t)\right|^{2}\right) \\
& \leqslant {\left[A F_{1}^{2} \eta^{2} M(\mathcal{D})+\mu_{0}+m_{1}(\mathcal{D})\left(1+2 H T_{1}^{2 H}-1\right)\right] \int_{0}^{\tau \wedge T_{1}} E\left|Z_{s}-Q_{s}+Q_{s}-K(a, s)\right|^{2} d s } \\
&+\left(\mu_{0}+1\right) \int_{0}^{\tau \wedge T_{1}} E\left|Q_{s}-K(a, s)\right|^{2} d s+8 m_{4}^{\frac{1}{2}}(\mathcal{D}) m_{5}^{\frac{1}{2}}(\mathcal{D}) L_{1} L_{2}^{H} \Delta t \\
& \leqslant {\left[2 A F_{1}^{2} \eta^{2} M(\mathcal{D})+3 \mu_{0}+1+2 m_{1}(\mathcal{D})\left(1+2 H T_{1}^{2 H}-1\right)\right] \int_{0}^{T_{1}} E\left(\sup _{0 \leqslant t \leqslant \tau \wedge T_{1}}\left|Q_{r}-K(a, r)\right|^{2}\right) d r } \\
&+2\left[A F_{1}^{2} \eta^{2} M(\mathcal{D})+\mu_{0}+m_{1}(\mathcal{D})\left(1+2 H T_{1}^{2 H-1}\right)\right] \int_{0}^{\tau \wedge T_{1}} E\left|Z_{s}-Q_{s}\right|^{2} d s \\
&+8 m_{4}^{\frac{1}{2}}(\mathcal{D}) m_{5}^{\frac{1}{2}}(\mathcal{D}) \mathrm{L}_{1} \mathrm{~L}_{2}^{H} \Delta t
\end{aligned}
$$

for all $t \in[0, T]$.

Applying Gronwall's inequality, Eq. (2.3) and expression $Z_{s}=Z(a, s)=Q\left(a,\left[\frac{s}{\Delta t}\right] \Delta t\right)$ (where $\left[\frac{s}{\Delta t}\right]$ is 
the integer part of $\frac{s}{\Delta t}$ ), we obtain a bound of the form

$$
\begin{aligned}
& \left|\mathrm{Z}_{\mathrm{s}}-\mathrm{Q}_{\mathrm{s}}\right|^{2}=\left|\mathrm{Q}\left(\mathrm{a},\left[\frac{\mathrm{s}}{\Delta \mathrm{t}}\right] \Delta \mathrm{t}\right)-\mathrm{Q}_{\mathrm{s}}\right|^{2} \\
& =\mid-\int_{0}^{\left[\frac{s}{\Delta \mathrm{t}}\right] \Delta \mathrm{t}} \frac{\partial Z_{\mathfrak{u}}}{\partial \mathrm{a}} \mathrm{du}+\int_{0}^{s} \frac{\partial Z_{\mathfrak{u}}}{\partial \mathrm{a}} \mathrm{du}-\mu_{0} \int_{0}^{\left[\frac{\mathrm{s}}{\Delta \mathrm{t}}\right] \Delta \mathrm{t}} \mathrm{Z}_{\mathfrak{u}} \mathrm{du}+\mu_{0} \int_{0}^{s} Z_{\mathfrak{u}} d u \\
& +\int_{0}^{\left[\frac{s}{\Delta \mathrm{t}}\right] \Delta \mathrm{t}} \mathrm{f}\left(\mathrm{u}, \mathrm{Z}_{\mathfrak{u}}\right) d u-\int_{0}^{s} f\left(u, Z_{\mathfrak{u}}\right) d u+\int_{0}^{\left[\frac{s}{\Delta \mathrm{t}}\right] \Delta \mathrm{t}} g\left(u, \mathrm{Z}_{\mathfrak{u}}\right) d B_{u}^{\mathrm{H}}-\left.\int_{0}^{s} g\left(u, Z_{\mathfrak{u}}\right) d B_{u}^{H}\right|^{2} \\
& \leqslant 4\left|\int_{\left[\frac{s}{\Delta t}\right] \Delta t}^{s} \frac{\partial Q\left(a,\left[\frac{s}{\Delta t}\right] \Delta t\right)}{\partial a} d u\right|^{2}+4\left|\int_{\left[\frac{s}{\Delta t}\right] \Delta t}^{s} \mu_{0} Q\left(a,\left[\frac{s}{\Delta t}\right] \Delta t\right) d u\right|^{2} \\
& +4\left|\int_{\left[\frac{s}{\Delta t}\right] \Delta t}^{s} f\left(u, Q\left(a,\left[\frac{s}{\Delta t}\right] \Delta t\right)\right) d u\right|^{2}+4\left|\int_{\left[\frac{s}{\Delta t}\right] \Delta t}^{s} g\left(u, Q\left(a,\left[\frac{s}{\Delta t}\right] \Delta t\right)\right) d B_{u}^{H}\right|^{2} .
\end{aligned}
$$

Applying the definition of $\mathrm{fBm}$ on the last term of (3.4) again, we have

$$
\left|E \int_{\left[\frac{s}{\Delta t}\right] \Delta t}^{s} g\left(u, Q\left(a,\left[\frac{s}{\Delta t}\right] \Delta t\right)\right) d B_{u}^{H}\right|^{2} \leqslant m_{4}(\mathcal{D}) T_{1}^{2 H} \Delta t
$$

Thus, by conditions (2.6) and (2.7) and inequality (3.4), for $\mathrm{T} \Delta \mathrm{t}<1$,

$$
\begin{aligned}
E \int_{0}^{\tau \wedge T_{1}}\left|Z_{s}-Q_{s}\right|^{2} d s & \leqslant 4 T_{1}\left[m_{6}(\mathcal{D})+\mu_{0}^{2} m_{5}(\mathcal{D})+m_{4}(\mathcal{D})\right] \Delta t^{2}+4 m_{4}(\mathcal{D}) T_{1}^{2 H} \Delta t \\
& \leqslant 4\left[m_{6}(\mathcal{D})+\mu_{0}^{2} m_{5}(\mathcal{D})+m_{4}(\mathcal{D})+m_{4}(\mathcal{D}) T_{1}^{2 H}\right] \Delta t
\end{aligned}
$$

Substituting (3.5) into (3.3), one can get

$$
E\left(\sup _{0 \leqslant t \leqslant \tau \wedge T_{1}}\left|Q_{t}-K(a, t)\right|^{2}\right) \leqslant C_{1}(\mathcal{D}) \Delta t+C_{2}(\mathcal{D}) \int_{0}^{T_{1}} E\left(\sup _{0 \leqslant t \leqslant \tau \wedge T_{1}}\left|Q_{r}-K(a, r)\right|^{2}\right) d r
$$

where

$$
\begin{aligned}
\mathrm{C}_{1}(\mathcal{D})= & 8\left\{\left[\mathrm{AF}_{1}^{2} \eta^{2} \mathrm{M}(\mathcal{D})+\mu_{0}+\mathrm{m}_{1}(\mathcal{D})\left(1+2 \mathrm{HT}_{1}^{2 \mathrm{H}-1}\right)\right] *\left[\mathrm{~m}_{6}(\mathcal{D})+\mu_{0}^{2} \mathrm{~m}_{5}(\mathcal{D})+\mathrm{m}_{4}(\mathcal{D})+\mathrm{m}_{4}(\mathcal{D}) \mathrm{T}_{1}^{2 \mathrm{H}}\right]\right. \\
& \left.+\mathrm{m}_{4}^{\frac{1}{2}}(\mathcal{D}) \mathrm{m}_{5}^{\frac{1}{2}}(\mathcal{D}) \mathrm{L}_{1} \mathrm{~L}_{2}^{\mathrm{H}}\right\} \\
\mathrm{C}_{2}(\mathcal{D})= & 2 A \mathrm{AF}_{1}^{2} \eta^{2} \mathrm{M}(\mathcal{D})+3 \mu_{0}+1+2 \mathrm{~m}_{1}(\mathcal{D})\left(1+2 \mathrm{HT}_{1}^{2 \mathrm{H}-1}\right) .
\end{aligned}
$$

On applying the Gronwall inequality we then have the following inequality

$$
E\left[\sup _{0 \leqslant \mathrm{t} \leqslant \tau \wedge T}\left|\mathrm{Q}_{\mathrm{t}}-\mathrm{K}(\mathrm{a}, \mathrm{t})\right|^{2}\right] \leqslant \mathrm{C}_{1}(\mathcal{D}) \mathrm{e}^{\mathrm{C}_{2}(\mathcal{D}) \mathrm{T}} \Delta \mathrm{t}=\mathrm{C}(\mathcal{D}) \Delta \mathrm{t}
$$

So, the proof is complete.

Theorem 3.2. If $\theta$ is the first exit time of the solution $\mathrm{K}(\mathrm{a}, \mathrm{t})$ to (2.1) from the domain $\mathcal{D}(\mathrm{r})$, and a function $\mathrm{V}(\mathrm{t}, \mathrm{K})$ exists which satisfies conditions (c) and (d), then the probability

$$
\mathrm{P}(\theta \geqslant \mathrm{T}) \geqslant 1-\varepsilon
$$


Proof. Assuming that there exists a non-negative function $\mathrm{V}(\mathrm{t}, \mathrm{K})$ satisfying condition $(\mathrm{c})$, and applying Itô's formula to $\mathrm{V}(\mathrm{t}, \mathrm{K})$, we have

$$
d V(t, K(a, t))=\operatorname{LV}(t, K(a, t))+V_{K}(t, K(a, t)) g(t, K(a, t)) d B_{t}^{H} .
$$

Taking integral over the interval $[0, \mathrm{t} \wedge \theta]$ and taking expectations, yields

$$
\mathrm{EV}(\mathrm{t} \wedge \theta, \mathrm{K}(\mathrm{a}, \mathrm{t} \wedge \theta))=\mathrm{V}\left(\mathrm{t}_{0}, \mathrm{~K}_{0}\right)+\mathrm{E} \int_{0}^{\mathrm{t} \wedge \theta} \operatorname{LV}(\mathrm{s}, \mathrm{K}(\mathrm{a}, \mathrm{s})) \mathrm{ds}
$$

By condition (d), it follows that

$$
\mathrm{EV}(\mathrm{t} \wedge \theta, \mathrm{K}(\mathrm{a}, \mathrm{t} \wedge \theta)) \leqslant \mathrm{V}\left(\mathrm{t}_{0}, \mathrm{~K}_{0}\right)+\mathrm{E} \int_{0}^{\mathrm{T}}\left(\mathrm{m}_{2}(\mathcal{D})+\psi_{1}(\mathrm{~s})+\psi_{2}(\mathrm{~s}) \mathrm{V}(\mathrm{s}, \mathrm{K}(\mathrm{a}, \mathrm{s})) \mathrm{ds}\right.
$$

Applying Gronwall's lemma, one can easily obtain that almost surely

$$
E V(t, K(a, t)) \leqslant\left(V\left(t_{0}, K_{0}\right)+m_{2}(\mathcal{D}) T+\int_{0}^{T} \psi_{1}(s) d s\right) \times \exp \left(\int_{0}^{T} \psi_{2}(s) d s\right)
$$

Let

$$
\mathrm{N}_{1}=\left(\mathrm{V}\left(\mathrm{t}_{0}, \mathrm{~K}_{0}\right)+\mathrm{m}_{2}(\mathcal{D}) \mathrm{T}+\int_{0}^{\mathrm{T}} \psi_{1}(\mathrm{~s}) \mathrm{ds}\right) \times \exp \left(\int_{0}^{\mathrm{T}} \psi_{2}(\mathrm{~s}) \mathrm{ds}\right) .
$$

Note that $V(\theta, K(a, \theta))=r$, and $K(a, \theta)$ is valued on the boundary of $\mathcal{D}(r)$, the probability $p(\theta<T)$ can be bounded as

$$
\mathrm{N}_{1} \geqslant \mathrm{E}\left[\mathrm{V}(\mathrm{t} \wedge \theta, \mathrm{K}(\mathrm{a}, \mathrm{t} \wedge \theta)] \geqslant \mathrm{E}\left[\mathrm{V}(\mathrm{t} \wedge \theta, \mathrm{K}(\mathrm{a}, \mathrm{t} \wedge \theta)) \mathrm{I}_{\{\theta<\mathrm{T}\}}(\omega)\right] \geqslant \operatorname{rE}\left[\mathrm{I}_{\{\theta<\mathrm{T}\}}(\omega)\right] \geqslant \operatorname{rP}(\theta<\mathrm{T})\right.
$$

Rearranging (3.6), we have

$$
P(\theta<T) \leqslant N_{1} / r=\varepsilon .
$$

Theorem 3.2 is proved.

We can obtain the following results deduced from Theorem 3.2 directly.

Lemma 3.3. Let $\theta$ be the first exit time of the solution $\mathrm{K}(\mathrm{a}, \mathrm{t})$ to $E q$. (1.1) from the domain $\mathcal{D}(\mathrm{r})$, and the coefficients of Eq. (1.1) satisfy condition (a). If there exists a function $\mathrm{V}(\mathrm{t}, \mathrm{K})$ satisfying conditions (c) and (d), then $\lim _{\mathrm{r} \rightarrow \infty} \mathcal{D}(\mathrm{r}) \equiv \mathrm{G}$ and, for any $\mathrm{t} \in[0, \mathrm{~T}]$ and $\mathrm{K}_{0} \in \mathrm{G}, \mathrm{K}(\mathrm{a}, \mathrm{t})$ remains in $\mathrm{G}$. Thus, for all finite $\mathrm{T}, \mathrm{K}(\mathrm{a}, \mathrm{t})$ is the unique solution to Eq. (1.1) on $\mathrm{t} \in[0, \mathrm{~T}]$.

Proof. The proof of this lemma is similar to that in [19].

For the Euler approximate solution $\mathrm{Q}_{\mathrm{t}}$, one can get the following Theorem 3.4 similarly.

Theorem 3.4. If $\rho$ is the first exit time of the Euler approximate solution $\mathrm{Q}_{\mathrm{t}}$ to Eq. (2.3) from the domain $\mathcal{D}(\mathrm{r})$, and if $\mathrm{f}(\mathrm{t}, \mathrm{K})$ and $\mathrm{g}(\mathrm{t}, \mathrm{K})$ satisfy condition $(\mathrm{b})$ and there exists a function $\mathrm{V}(\mathrm{t}, \mathrm{K}(\mathrm{t}, \mathrm{a}))$ satisfying conditions $(\mathrm{c})-(\mathrm{e})$, then

$$
\mathrm{P}(\rho \geqslant \mathrm{T}) \geqslant 1-\varepsilon(1+\overline{\mathrm{N}}(\mathcal{D}) \Delta \mathrm{t})
$$

for sufficiently small $\Delta \mathrm{t}$, where $\overline{\mathrm{N}}(\mathcal{D})=\mathrm{N}(\mathcal{D}) e^{-\int_{0}^{\mathrm{T}} \psi_{2}(\mathrm{~s}) \mathrm{ds}} /\left(\mathrm{V}\left(0, \mathrm{~K}_{0}\right)+\int_{0}^{\mathrm{T}} \psi_{1}(\mathrm{~s}) \mathrm{ds}\right)$. 
Proof. Note that $\mathrm{Q}_{\mathrm{t}}$ is also the solution to Eq. (2.4), and applying Itô's formula to $\mathrm{V}\left(\mathrm{t}, \mathrm{Q}_{\mathrm{t}}\right)$ yields,

$$
\begin{aligned}
d V\left(t, Q_{t}\right)= & V_{t}\left(t, Q_{t}\right) d t+\left\langle V_{K}\left(t, Q_{t}\right),-\frac{\partial Z_{t}}{\partial a}-\mu(t, a) Z_{t}+f\left(t, Z_{t}\right)\right\rangle d t \\
& +\frac{1}{2} H t^{2 H-1} V_{K K}\left(t, Q_{t}\right)\left\|g\left(t, Z_{t}\right)\right\|_{2}^{2} d t+\left\langle V_{K}\left(t, Q_{t}\right), g\left(t, Z_{t}\right) d B_{t}^{H}\right\rangle \\
= & L V\left(Z_{t}\right)+\left(V_{t}\left(t, Q_{t}\right)-V_{t}\left(t, Z_{t}\right) d t+\left\langle V_{K}\left(t, Q_{t}\right)-V_{K}\left(t, Z_{t}\right),-\frac{\partial Z_{t}}{\partial a}-\mu(t, a) Z_{t}+f\left(t, Z_{t}\right)\right\rangle d t\right. \\
& +\frac{1}{2} H t^{2 H-1}\left(V_{K K}\left(t, Q_{t}\right)-V_{K K}\left(t, Z_{t}\right)\right)\left\|g\left(t, Z_{t}\right)\right\|_{2}^{2} d t+\left\langle V_{K}\left(t, Q_{t}\right), g\left(t, Z_{t}\right) d B_{t}^{H}\right\rangle .
\end{aligned}
$$

Applying condition (d), we have

$$
\begin{aligned}
d V\left(t, Q_{t}\right) \leqslant & m_{2}(\mathcal{D})+\psi_{1}(t)+\psi_{2}(t) V\left(t, Z_{t}\right)+\left[V_{t}\left(t, Q_{t}\right)-V_{t}\left(t, Z_{t}\right)\right] d t \\
& +\left\langle V_{K}\left(t, Q_{t}\right)-V_{K}\left(t, Z_{t}\right),-\frac{\partial Z_{t}}{\partial a}-\mu(t, a) Z_{t}+f\left(t, Z_{t}\right)\right\rangle d t \\
& +\frac{1}{2} H t^{2 H-1}\left(V_{K K}\left(t, Q_{t}\right)-V_{K K}\left(t, Q_{t}\right)\right)\left\|g\left(t, Z_{t}\right)\right\|_{2}^{2} d t+\left\langle V_{K}\left(t, Q_{t}\right), g\left(t, Z_{t}\right) d B_{t}^{H}\right\rangle \\
\leqslant & m_{2}(\mathcal{D})+\psi_{1}(t)+\psi_{2}(t) V\left(t, Q_{t}\right)+\psi_{2}(t)\left[V\left(t, Z_{t}\right)-V\left(t, Q_{t}\right)\right] d t \\
& +\left(V_{t}\left(t, Q_{t}\right)-V_{t}\left(t, Z_{t}\right)\right) d t \\
& \left.+\left\langle V_{K}\left(t, Q_{t}\right)-V_{K}\left(t, Z_{t}\right),-\frac{\partial Z_{t}}{\partial a}-\mu(t, a) Z_{t}\right)+f\left(t, Z_{t}\right)\right\rangle d t \\
& +\frac{1}{2} H t^{2 H-1}\left(V_{K K}\left(t, Q_{t}\right)-V_{K K}\left(t, Z_{t}\right)\right)\left\|g\left(t, Z_{t}\right)\right\|_{2}^{2} d t+\left\langle V_{K}\left(t, Q_{t}\right), g\left(t, Z_{t}\right) d B_{t}^{H}\right\rangle .
\end{aligned}
$$

Taking integral over the interval $[0, \rho \wedge t]$ and taking expectations, yields

$$
\begin{aligned}
E\left[V\left(\rho \wedge t, Q_{\rho \wedge t}\right)\right] \leqslant & V\left(0, K_{0}\right)+m_{2}(\mathcal{D}) T+\int_{0}^{\rho \wedge t} \psi_{1}(s) d s+E \int_{0}^{\rho \wedge t} \psi_{2}(s) V\left(s, Q_{s}\right) d s \\
& +E \int_{0}^{\rho \wedge t}\left(V_{t}\left(s, Q_{s}\right)-V_{t}\left(s, Z_{s}\right) d s+E \int_{0}^{\rho \wedge t} \psi_{2}(s)\left[V\left(s, Z_{s}\right)-V\left(s, Q_{s}\right)\right] d s\right. \\
& +E \int_{0}^{\rho \wedge t}\left\langle V_{K}\left(s, Q_{s}\right)-V_{K}\left(s, Z_{s}\right),-\frac{\partial Z_{s}}{\partial a}-\mu(s, a) Z_{s}+f\left(s, Z_{s}\right)\right\rangle d s \\
& +\frac{1}{2} H E \int_{0}^{\rho \wedge t} s^{2 H-1}\left(V_{K K}\left(s, Q_{s}\right)-V_{K K}\left(s, Z_{s}\right)\right)\left\|g\left(s, Z_{s}\right)\right\|_{2}^{2} d s \\
\leqslant & V\left(0, K_{0}\right)+m_{2}(\mathcal{D}) T+\int_{0}^{\rho \wedge t} \psi_{1}(s) d s+E \int_{0}^{\rho \wedge t} \psi_{2}(s) V\left(s, Q_{s}\right) d s \\
& +E \int_{0}^{\rho \wedge t} \psi_{2}(s)\left|V\left(s, Z_{s}\right)-V\left(s, Q_{s}\right)\right| d s+E \int_{0}^{\rho \wedge t} \mid V_{t}\left(s, Q_{s}\right)-V_{t}\left(s, Z_{s} \mid d s\right. \\
& +E \int_{0}^{\rho \wedge t}\left|V_{K}\left(s, Q_{s}\right)-V_{K}\left(s, Z_{s}\right)\right|\left|-\frac{\partial Z_{s}}{\partial a}-\mu(s, a) Z_{s}+f\left(s, Z_{s}\right)\right| d s \\
& +\frac{1}{2} H E \int_{0}^{\rho \wedge t} s^{2 H-1}\left|V_{K K}\left(s, Q_{s}\right)-V_{K K}\left(s, Z_{s}\right)\right|\left\|g\left(s, Z_{s}\right)\right\|_{2}^{2} d s .
\end{aligned}
$$

Therefore, applying inequalities (2.5)-(2.7) and condition (e), we get that

$$
\begin{aligned}
E\left[V\left(\rho \wedge t, Q_{\rho \wedge t}\right)\right] \leqslant & V\left(0, K_{0}\right)+m_{2}(\mathcal{D}) T+\int_{0}^{T} \psi_{1}(s) d s \\
& +N_{2}(\mathcal{D}) m_{3}(\mathcal{D}) E \int_{0}^{\rho \wedge t}\left|Z_{s}-Q_{s}\right| d s+E \int_{0}^{t} \psi_{2}(s) V\left(\rho \wedge s, Q_{\rho \wedge s}\right) d s
\end{aligned}
$$


where

$$
\mathrm{N}_{2}(\mathcal{D})=\mathrm{m}_{4}^{1 / 2}(\mathcal{D})+\mu_{0} \mathrm{~m}_{5}^{1 / 2}(\mathcal{D})+\mathrm{m}_{6}^{1 / 2}(\mathcal{D})+\mathrm{m}_{4}(\mathcal{D}) \mathrm{T}^{2 \mathrm{H}-1} / 2+\sup _{0 \leqslant \mathrm{t} \leqslant \mathrm{T}} \psi_{2}(\mathrm{t})+1 .
$$

Applying Hölder inequality and (3.5), for all $t \in[0, T]$, we have the bound

$$
\int_{0}^{\rho \wedge t} E\left|Z_{s}-Q_{s}\right| d s \leqslant\left(4 T\left(m_{6}(\mathcal{D})+\mu_{0}^{2} m_{5}(\mathcal{D})+m_{4}(\mathcal{D})+m_{4}(\mathcal{D}) T^{2 H}\right)\right)^{1 / 2} \Delta t^{1 / 2}
$$

Thus

$$
\begin{aligned}
E\left[V\left(\rho \wedge t, Q_{\rho \wedge t}\right)\right] \leqslant & V\left(0, K_{0}\right)+m_{2}(\mathcal{D}) T+\int_{0}^{T} \psi_{1}(s) d s \\
& +N_{2}(\mathcal{D}) m_{3}(\mathcal{D})\left(4 T\left(m_{6}(\mathcal{D})+\mu_{0}^{2} m_{5}(\mathcal{D})+m_{4}(\mathcal{D})+m_{4}(\mathcal{D}) T^{2 H}\right)\right)^{1 / 2} \triangle t^{1 / 2} \\
& +\int_{0}^{t} \psi_{2}(s) E V\left(\rho \wedge s, Q_{\rho \wedge s}\right) d s .
\end{aligned}
$$

Applying the Gronwall inequality, yields

$$
E\left[V\left(\rho \wedge t, Q_{\rho \wedge t}\right)\right] \leqslant\left[V\left(0, K_{0}\right)+m_{2}(\mathcal{D}) T+\int_{0}^{T} \psi_{1}(s) d s\right] e^{\int_{0}^{T} \psi_{2}(s) d s}+N(\mathcal{D}) \Delta t^{1 / 2},
$$

where $N(\mathcal{D})=e^{\int_{0}^{T}} \psi_{2}(s) d s N_{2}(\mathcal{D}) m_{3}(\mathcal{D})\left\{4 T\left[m_{6}(\mathcal{D})+\mu_{0}^{2} m_{5}(\mathcal{D})+m_{4}(\mathcal{D})+m_{4}(\mathcal{D}) T^{2 H}\right]\right\}^{1 / 2}$.

A similar argument, being used to prove Theorem 3.2, can be used to bound $P(\rho<T)$. Since $Q_{\rho}$ is valued on the boundary of $\mathcal{D}(r)$ then $V\left(\rho, Q_{\rho}\right)=r$, one can get

$$
\begin{aligned}
{\left[\mathrm{V}\left(0, \mathrm{~K}_{0}\right)+\int_{0}^{\mathrm{T}} \psi_{1}(\mathrm{~s}) \mathrm{ds}\right] \mathrm{e}^{\int_{0}^{\mathrm{T}} \psi_{2}(s) \mathrm{ds}}+\mathrm{N}(\mathcal{D}) \triangle \mathrm{t} } & \geqslant \mathrm{E}\left[\mathrm{V}\left(\mathrm{T} \wedge \rho, \mathrm{Q}_{\mathrm{T} \wedge \rho}\right]\right. \\
& \geqslant \mathrm{E}\left[\mathrm{V}\left(\mathrm{T} \wedge \rho, \mathrm{Q}_{\mathrm{T} \wedge \rho}\right) \mathrm{I}_{\{\rho<\mathrm{T}\}}(\omega)\right] \\
& \geqslant \operatorname{rE}\left[\mathrm{I}_{\{\rho<\mathrm{T}\}}(\omega)\right] \geqslant \operatorname{rP}(\rho<\mathrm{T}) .
\end{aligned}
$$

Rearranging the inequality and defining $\bar{N}(\mathcal{D})=\mathrm{N}(\mathcal{D}) e^{-\int_{0}^{T} \psi_{2}(s) \mathrm{d} s} /\left(\mathrm{V}\left(0, \mathrm{~K}_{0}\right)+\int_{0}^{\mathrm{T}} \psi_{1}(\mathrm{~s}) \mathrm{d} s\right)$, and defining $\varepsilon=\frac{\mathrm{N}_{1}}{\mathrm{r}} \in(0,1)$ as in (3.7), we have

$$
P(\rho<T) \leqslant\left(1+\bar{N}(\mathcal{D}) \triangle t^{1 / 2}\right) \varepsilon .
$$

Theorems 3.2 and 3.4 show that both $K(a, t)$ and $Q_{t}$ remain in the domain $\mathcal{D}(r)$. And therefore, by Theorem 3.1, the Euler approximate solution $Q_{t}$ will converge to the $K(a, t)$ with probability

$$
\mathrm{P}(\tau<\mathrm{T}) \leqslant \mathrm{P}(\rho<\mathrm{T})+\mathrm{P}(\mathrm{s}<\mathrm{T}) \leqslant\left(2+\overline{\mathrm{N}}(\mathcal{D}) \Delta \mathrm{t}^{1 / 2}\right) \varepsilon
$$

Theorem 3.5. Let $\mathrm{G}$ be an open subset of $\mathrm{V}$, and denote the unique solution to Eq. (1.1) for $\mathrm{t} \in[0, \mathrm{~T}]$ with initial value $\mathrm{K}_{0} \in \mathrm{G}$ by $\mathrm{K}(\mathrm{a}, \mathrm{t}) \in \mathrm{G}$. Let $\mathrm{Q}_{\mathrm{t}}$ be the Euler approximate solution to (2.2) and also let $\mathcal{D} \subseteq \mathrm{G}$ be any compact set. Suppose conditions (a)-(e) are satisfied, and for all $a \in(0, A), \sup _{t \in \mathcal{D}}\left|\frac{Q_{t}-K(a, t)}{Z_{t}-K(a, t)}\right|$ exists. Then for any $\varepsilon, \delta>0$ there exists $\Delta \mathrm{t}^{*}>0$ such that (when $\Delta \mathrm{t} \leqslant \Delta \mathrm{t}^{*}$ )

$$
P\left(\sup _{0 \leqslant t \leqslant T}\left|Q_{t}-K(a, t)\right|^{2} \geqslant \delta\right) \leqslant \varepsilon
$$


Proof. Introducing the event sub-space $\bar{\Omega}=\left\{\omega: \sup _{0 \leqslant t \leqslant T}\left|Q_{t}-K(a, t)\right|^{2} \geqslant \delta\right\}$ and using Theorem 3.1, we find that

$$
\begin{aligned}
C(\mathcal{D}) \Delta t & \geqslant E\left[\sup _{0 \leqslant t \leqslant T}\left|Q_{t}-K(a, t)\right|^{2}\right] \\
& \geqslant E\left[I_{\{\tau \geqslant T\}}(\omega) \sup _{0 \leqslant t \leqslant T}\left|Q_{t}-K(a, t)\right|^{2}\right] \\
& \geqslant E\left[I_{\{\tau \geqslant T\}}(\omega) I_{\{\bar{\Omega}\}} \sup _{0 \leqslant t \leqslant T}\left|Q_{t}-K(a, t)\right|^{2}\right] \\
& \geqslant \delta E\left[I_{\{\tau \geqslant T\}}(\omega) I_{\{\bar{\Omega}\}}(\omega)\right]=\delta P((\tau \geqslant T) \bigcap \bar{\Omega}) \geqslant \delta[P(\bar{\Omega})-P(\tau<T)] .
\end{aligned}
$$

Whence on using (3.8) we conclude that probability

$$
K(a, \bar{\Omega})=P\left(\sup _{0 \leqslant t \leqslant T}\left|Q_{t}-K(a, t)\right|^{2} \geqslant \delta\right) \leqslant 2 \epsilon+\epsilon \bar{N}((D)) \Delta t^{1 / 2}+\frac{C((D))}{\delta} \Delta t,
$$

which, for appropriate choice of $\Delta t$, proves Theorem 3.5.

\section{An example}

We consider the following stochastic age-dependent capital system with respect to fBm

$$
\begin{cases}\frac{\partial K(a, t)}{\partial t}+\frac{\partial K(a, t)}{\partial a}=-\frac{1}{(1-a)^{2}} K(a, t)-t K(a, t)+K(a, t) \frac{d B_{t}^{H}}{d t}, & (a, t) \in Q \\ K(a, 0)=\exp \left(-\frac{1}{1-a}\right), & a \in[0,1], \\ K(0, t)=\gamma(t) A(t) \frac{1}{(2-t)^{2}} \int_{0}^{1} K(a, t) d a, & t \in[0, T] \\ N(t)=\int_{0}^{1} K(a, t) d a, & t \in[0, T]\end{cases}
$$

where $\mathbf{Q}=(0,1) \times(0, T) . B_{t}^{H}$ is a $\mathrm{fBm}$ with Hurst number $\mathrm{H}=\frac{3}{4}$. For the simulation of $B_{t}^{H}$ with $H=3 / 4$, the generators of the $\mathrm{fBm}$ described in [1] are used. We can set this problem in our formulation by taking $\mathbb{H}=\mathrm{L}^{2}([0,1])$ and $\mathrm{V}=\mathrm{B}_{0}^{1}([0,1])$ (a Sobolev space with elements satisfying the boundary conditions above), and set $\mu(t, a)=\frac{1}{(1-a)^{2}}, \gamma(t) A(t)=2 t, F(L(t), N(t))=\frac{1}{(2-t)^{2}} \int_{0}^{A} K(a, t) d a, L(t)=\frac{1}{(2-t)^{2}}, f(t, K)=$ $-\mathrm{tK}, \mathrm{g}(\mathrm{t}, \mathrm{K})=\mathrm{K}$, and $\mathrm{K}(\mathrm{a}, 0)=\exp \left(-\frac{1}{1-\mathrm{a}}\right)$.

Obviously, $F(L, N)$ and $\mu(t, a)$ satisfy condition (a) and the operators $f$ and $g$ satisfy condition (b). Then, the approximate solution will converge to the true solution of Eq. (4.1) for any $(a, t) \in \mathbf{Q}$ in the sense of Theorem 3.5. Here we take $T=1$ in Eq. (4.1). The results are obtained with fixed step sizes $\Delta \mathrm{t}=0.005, \Delta \mathrm{a}=0.05$ in our calculation.

Figure 1 (a) shows the explicit solution to Eq. (4.1) without perturbation, that is $\operatorname{EK}(a, t)=\exp \left(-\frac{1}{1-a}-\right.$ $\frac{\mathrm{t}^{2}}{2}$ ). Figures 1 (b)-(d) show Euler approximate solution of Eq. (4.1) with 100, 1000, and 10000 experiments, respectively, where $\operatorname{EQ}(a, t)=\frac{1}{n} \sum_{k=1}^{n} K_{k}(a, t)$. These figures reveal the fact that the numerical approximate solution will tend to the true one in the mean sense.

For Eq. (4.1), it is difficult to obtain the analytic (explicit) solution, so the explicit solution $K(a, t)$ to Eq. (4.1) can be replaced by $\exp \left(-\frac{1}{1-a}-\frac{t^{2}}{2}\right)\left(1+\Delta B_{t}^{H}\right)$. Figures 2 (a) and (b) give the simulation of $\exp \left(-\frac{1}{1-a}-\frac{t^{2}}{2}\right)\left(1+\Delta B_{t}^{H}\right)$ and the Euler approximate solution $Q(a, t)$, respectively. The absolutely error and square error are also respectively shown in Figures 2 (c) and (d). The maximum of square error is not greater than 0.02. It is obvious that the numerical approximation will tend to the true solution in the mean square sense. 
(a) Analysis Solution without Perturbation

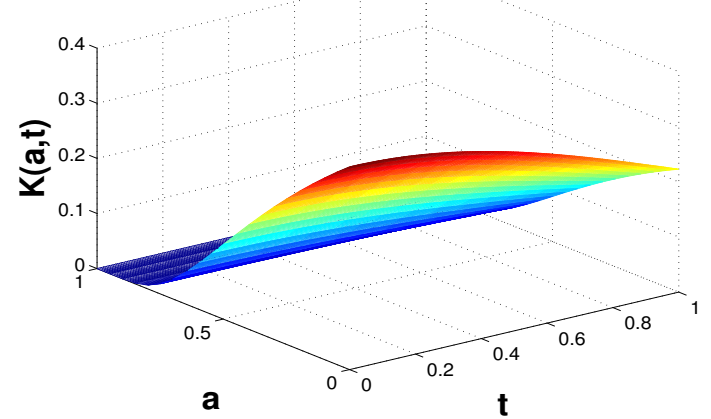

(c) Numerical Solution with 1000 Test

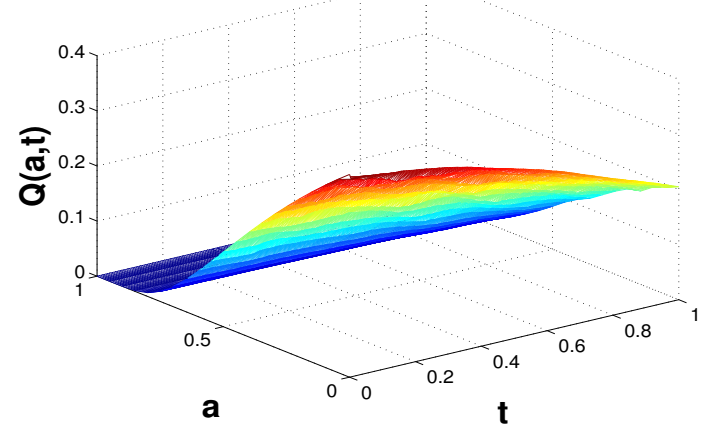

(b) Numerical Solution with 100 Test

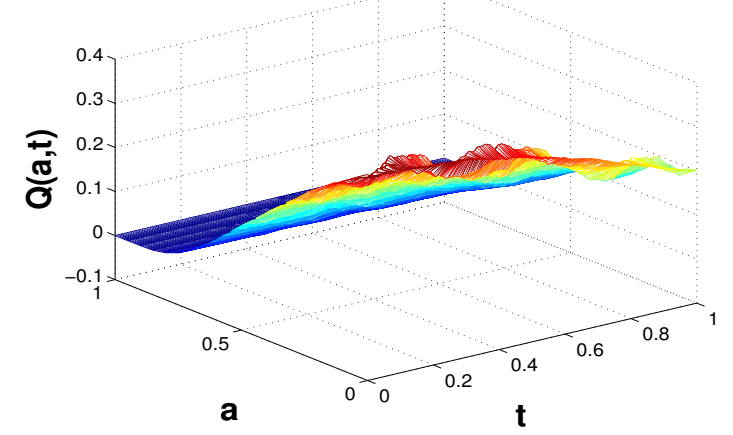

(d) Numerical Solution with 10000 Test

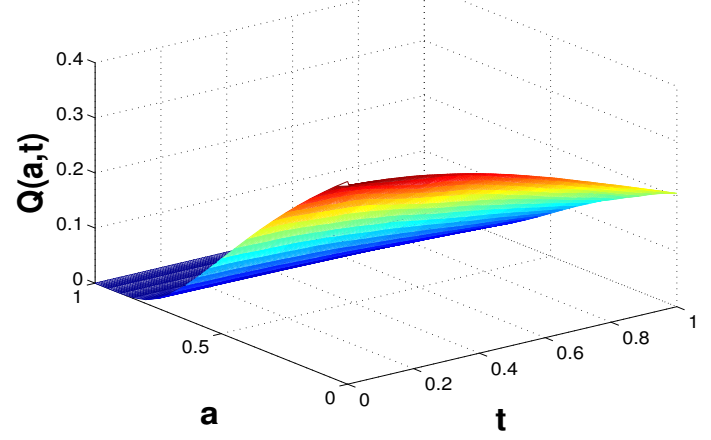

Figure 1: Numerical simulation of stochastic age-dependent capital system: (a) analysis solution and Euler approximate solution for several tests, (b) $n=100$, (c) $n=1000$, (d) $n=10000$.

(a) $\exp \left(-1 /(1-a)-t^{2} / 2\right)+$ Perturbation

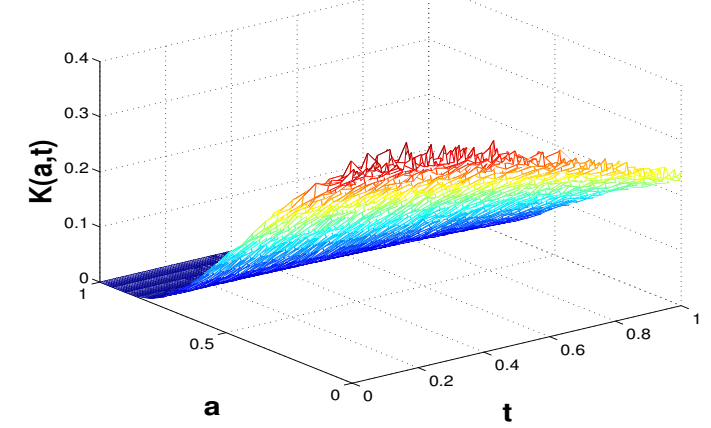

(c) Error

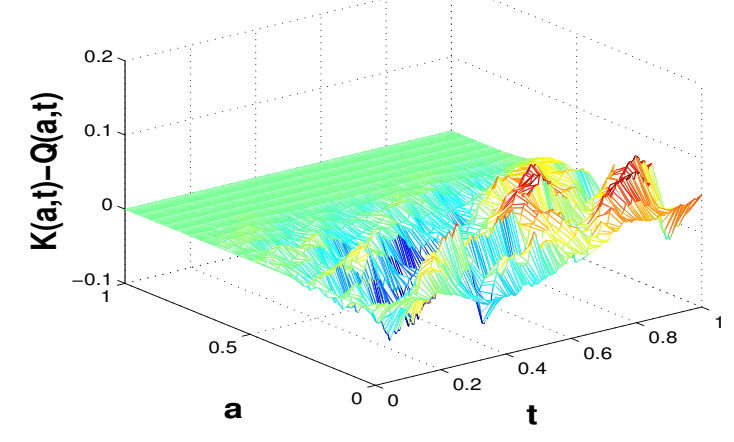

(b) Euler Numerical Solution

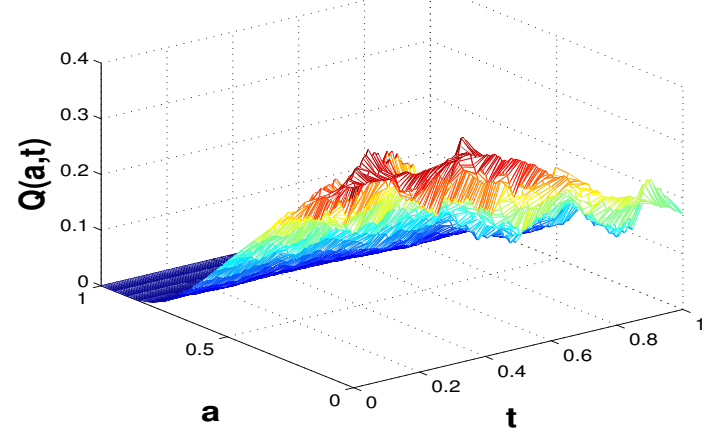

(d) Square Error

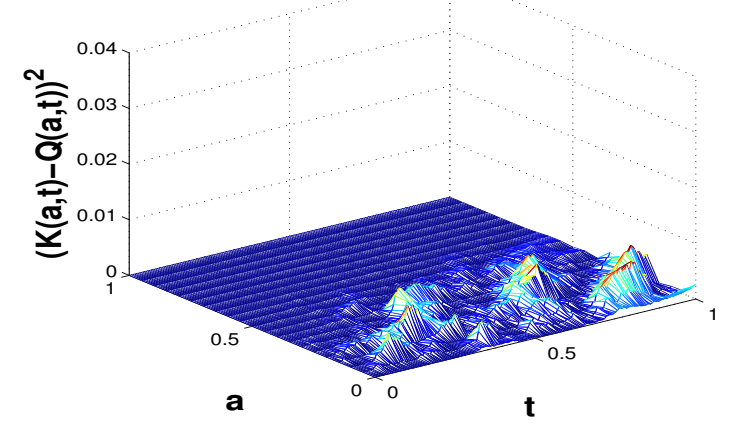

Figure 2: Error simulation of stochastic age-dependent capital system with fBm. 


\section{Acknowledgment}

This work was supported by the National Natural Science Foundation of China under Grants 11662016 and 11661064, by the Natural Science Foundation of Ningxia Hui Autonomous Region (China) under Grants NZ16005 and NZ14048, and supported in part by the Scientific Research Foundation of the Higher Education Institutions of Ningxia Hui Autonomous Region (China) under Grant NGY2016061.

\section{References}

[1] J. F. Coeurjolly, Simulation and identification of the fractional Brownian motion: a bibliographical and comparative study, J. Stat. Softw., 5 (2000), 1-53. 4

[2] Q.-H. Du, C.-L. Wang, Convergence analysis of semi-implicit Euler methods for solving stochastic age-dependent capital system with variable delays and random jump magnitudes, Math. Probl. Eng., 2014 (2014), 12 pages. 1

[3] T. E. Duncan, B. Maslowski, B. Pasik-Duncan, Stochastic equations in Hilbert space with a multiplicative fractional Gaussian noise, Stochastic Process. Appl., 115 (2005), 1357-1383. 3

[4] G. Feichtinger, R. F. Hartl, P. M. Kort, V. M. Veliov, Anticipation effects of technological progress on capital accumulation: a vintage capital approach, J. Econom. Theory, 126 (2006), 143-164. 1, 1

[5] G. Feichtinger, R. F. Hartl, P. M. Kort, V. M. Veliov, Vladimir, Capital accumulation under technological progress and learning: a vintage capital approach, Eur. J. Oper. Res., 172 (2006), 293-310. 1

[6] R. U. Goetz, N. Hritonenko, Y. Yatsenko, The optimal economic lifetime of vintage capital in the presence of operating costs, technological progress, and learning, J. Econom. Dynam. Control, 32 (2008), 3032-3053. 1, 1

[7] H. Gu, J.-R. Liang, Y.-X. Zhang, Time-changed geometric fractional Brownian motion and option pricing with transaction costs, Phys. A, 391 (2012), 3971-3977. 1

[8] K. Jańczak-Borkowska, Generalized BSDEs driven by fractional Brownian motion, Statist. Probab. Lett., 83 (2013), 805-811. 1

[9] Y.-M. Jiang, X.-C. Wang, Y.-J. Wang, On a stochastic heat equation with first order fractional noises and applications to finance, J. Math. Anal. Appl., 396 (2012), 656-669. 1

[10] P. E. Kloeden, E. Platen, Numerical solution of stochastic differential equations, Applications of Mathematics (New York), Springer-Verlag, Berlin, (1992). 1

[11] W.-J. Ma, Q.-M. Zhang, C.-Z. Han, Numerical analysis for stochastic age-dependent population equations with fractional Brownian motion, Commun. Nonlinear Sci. Numer. Simul., 17 (2012),1884-1893. 1

[12] A. Rathinasamy, Split-step $\theta$-methods for stochastic age-dependent population equations with Markovian switching, Nonlinear Anal. Real World Appl., 13 (2012), 1334-1345. 1, 1

[13] L. Ronghua, P. Wan-kai, L. Ping-kei, Convergence of numerical solutions to stochastic age-structured population equations with diffusions and Markovian switching, Appl. Math. Comput., 216 (2010), 744-752. 1, 1

[14] S. Rostek, R. Schöbel, A note on the use of fractional Brownian motion for financial modeling, Econ. Model., 30 (2013), 30-35. 1

[15] J. Wang, J.-R. Liang, L.-J. Lv, W.-Y. Qiu, F.-Y. Ren, Continuous time Black-Scholes equation with transaction costs in subdiffusive fractional Brownian motion regime, Phys. A, 391 (2012), 750-759. 1

[16] W.-L. Xiao, W.-G. Zhang, X.-L. Zhang, Y.-L. Wang, Pricing currency options in a fractional Brownian motion with jumps, Econ. Model., 27 (2010), 935-942.

[17] W.-L. Xiao, W.-G. Zhang, X.-L. Zhang, X.-L. Zhang, Pricing model for equity warrants in a mixed fractional Brownian environment and its algorithm, Phys. A, 391 (2012), 6418-6431. 1

[18] Q.-M. Zhang, Exponential stability of numerical solutions to a stochastic age-structured population system with diffusion, J. Comput. Appl. Math., 220 (2008), 22-33. 1

[19] Q.-M. Zhang, C.-Z. Han, Numerical analysis for stochastic age-dependent population equations, Appl. Math. Comput., 169 (2005), 278-294. 1, 3

[20] Q.-M. Zhang, Y.-T. Liu, X.-N. Li, Strong convergence of split-step backward Euler method for stochastic age-dependent capital system with Markovian switching, Appl. Math. Comput., 235 (2014), 439-453. 1

[21] Q.-M. Zhang, W.-K. Pang, P.-K. Leung, Exponential stability of numerical solutions for a class of stochastic age-dependent capital system with Poisson jumps, J. Comput. Appl. Math., 235 (2011), 3369-3377. 1

[22] Q.-M. Zhang, A. Rathinasamy, Convergence of numerical solutions for a class of stochastic age-dependent capital system with random jump magnitudes, Appl. Math. Comput., 219 (2013), 7297-7305. 1 